\title{
Chiral low-energy couplings from lattice computations in the epsilon regime
}

\section{Silvia Necco}

CERN, Physics Department

CH-1211 Geneva 23, Switzerland

E-mail: Silvia.Necco@cern.ch

\begin{abstract}
Lattice simulations in the $\varepsilon$-regime provide useful informations about the low-energy properties of QCD. In particular, they allow to extract the Low Energy Couplings from physical observables which are subject to different systematic corrections with respect to the usual infinite-volume situation. After a brief introduction on general properties of the $\varepsilon$-expansion in the chiral effective theory, the possible issues concerning lattice simulations are discussed. Finally, the matching of lattice results with the theoretical predictions and the extraction of the LO couplings $F$ and $\Sigma$ is presented.
\end{abstract}

6th International Workshop on Chiral Dynamics

July 6-10 2009

Bern, Switzerland 


\section{Introduction}

Lattice QCD simulations with light quarks are now approaching the domain of validity of the chiral effective theory. The matching of the lattice results with the effective theory predictions provides a determination of the Low Energy Couplings (LECs) from first principles. Many results have been presented in the past months for the leading order (LO) and next-to-leading order (NLO) couplings, both for the $N_{f}=2$ and $N_{f}=3$ effective theory (see [1] for a recent review). The main issue in this context is the control over the systematic uncertainties, namely the discretization errors, renormalization uncertainties, finite volume effects, the neglected higher order contributions in the chiral effective theory. For this reason, it is very useful to extract the LECs from a large set of observables and from different kinematical regimes: this will serve as a valuable cross-check between lattice determinations and will help to get a comprehensive picture of low-energy properties of QCD.

An interesting approach is to investigate QCD in a finite volume $V=L^{3} T$ in the so-called $\varepsilon$-regime $[2,3]$, where the chiral limit is approached by keeping $\mu=m \Sigma V \lesssim O(1)$, with $m$ the quark mass and $\Sigma$ the chiral condensate. This corresponds to the "unphysical" situation where the pion wavelength is larger than the size of the box, $M_{\pi} L<1$. The peculiar feature of this setup is that the pion zero-modes contribution becomes non-perturbative and must be treated exactly. This implies a reorganization of the chiral expansion, such that volume effects are enhanced (polynomial) while mass effects are suppressed with respect to the usual infinite-volume case (or $p$-regime, where $\left.M_{\pi} L \gg 1\right)$. For this reason, at a given order in the perturbative expansion, less LECs will appear: predictions are less "contaminated" by higher order unknown couplings, making the $\varepsilon$-regime potentially convenient for the determination of the LO constants $F$ and $\Sigma$.

The power counting adopted in the $\varepsilon$-expansion is the following:

$$
m \sim \varepsilon^{4}, \quad L^{-1}, T^{-1} \sim \varepsilon .
$$

The LO Euclidean chiral Lagrangian for $N_{f}$ degenerate flavors of mass $m$ is written as usual

$$
\mathscr{L}_{2}=\frac{F^{2}}{4} \operatorname{Tr}\left(\partial_{\mu} U \partial_{\mu} U^{\dagger}\right)-\frac{m \Sigma}{2} \operatorname{Tr}\left(U^{\dagger}+U\right) .
$$

The pseudo Nambu-Goldstone boson field $U \in S U\left(N_{f}\right)$ is parametrized as

$$
U(x)=U_{0} \exp \left(\frac{2 i}{F} \xi(x)\right),
$$

where $U_{0}$ represents the collective zero-mode, and the non-zero modes $\xi$ are still treated in perturbation theory, with $\xi \sim \varepsilon$. Then the LO partition function is given by

$$
\mathscr{Z}=\int_{S U\left(N_{f}\right)}\left[d U_{0}\right] \int[d \xi] \exp \left\{\frac{1}{2} \int_{V} d^{4} x \operatorname{Tr}\left(\partial_{\mu} \xi \partial_{\mu} \xi\right)+\frac{\mu}{2} \operatorname{Tr}\left(U_{0}+U_{0}^{\dagger}\right)\right\} .
$$

In the $\varepsilon$-regime, topology plays a relevant rôle [4]: by introducing a $\theta$ vacuum and Fourier transforming, one can define the partition function at fixed values of the topological charge $v$ :

$$
\mathscr{Z}_{v}=\int_{U\left(N_{f}\right)}\left[d U_{0}\right]\left(\operatorname{det} U_{0}\right)^{v} \int[d \xi] \exp \left\{\frac{1}{2} \int_{V} d^{4} x \operatorname{Tr}\left(\partial_{\mu} \xi \partial_{\mu} \xi\right)+\frac{\mu}{2} \operatorname{Tr}\left(U_{0}+U_{0}^{\dagger}\right)\right\} .
$$


From the partition function one can immediately obtain the quark condensate

$$
\Sigma(\mu)=\frac{\Sigma}{N_{f}} \frac{\partial \mathscr{Z}}{\partial \mu} \sim \Sigma \mu .
$$

As expected, $\Sigma(\mu) \rightarrow 0$ for $\mu \rightarrow 0$, since there is no spontaneous symmetry breaking in a finite box. Nevertheless, in the $\varepsilon$-regime one can extract informations about spontaneous symmetry breaking in infinite volume by means of a finite-size scaling.

\section{Lattice QCD in the epsilon regime}

Lattice simulations in the $\varepsilon$-regime are challenging, since one still needs a fairly large volume $(L \gg 1 /(4 \pi F))$, while the quark mass must be very small $(m \Sigma V \lesssim 1)$. This fact strongly influences the choice of the lattice action to be used. Ginsparg-Wilson (GW) fermions [5] are the best choice from the theoretical point of view, since they guarantee exact chiral symmetry at finite lattice spacing [6]. The Dirac spectrum is bounded from below, and arbitrarily small quark masses are then accessible in numerical simulations. Moreover, the topological charge has a natural definition through the index theorem: this means that one can perform simulations at fixed topological charge and match the lattice results with the corresponding predictions of the chiral effective theory for each topological sector. The most adopted implementations of Dirac operators satisfying the GW relation are the Neuberger Dirac operator [7], Domain Wall Dirac operator with infinite fifth dimension [8], the fixed-point Dirac operator [9]. The price to pay to maintain continuum-like chiral symmetry is the high computational cost. While a large number of quenched simulations has been performed using various implementations of the GW fermions [10, 11, 12, 13, 14, 15, 16, 17], dynamical simulations are still very challenging.

The possibility of simulating Wilson fermions in the $\varepsilon$-regime has not been considered for a long time: since chiral symmetry is explicitly broken at finite lattice spacing by the Wilson term, the spectral gap is not bounded from below, which makes the simulation of the small quark mass region rather problematic. At small quark masses the probability distribution of the gap can approach zero values, leading to possible integration instabilities and sampling inefficiencies in the algorithm. In $[18,19]$ the empirical stability bound $m>m_{\text {min }}$, with $m_{\min } \propto a / \sqrt{V}$ has been established. A possible solution to this problem comes from a reweighting technique [20] (see also [21]). This method has been applied in [22] to simulate Wilson fermions in the $\varepsilon$-regime. Lattice simulations in the $\varepsilon$-regime are feasible also with Wilson Twisted Mass fermions, as presented in [23, 24]. Another consequence of the explicit breaking of chiral symmetry with Wilson-like fermions is that an exact definition for the topological charge does not exist at nonzero lattice spacing. Lattice results nevertheless can be matched with ChPT predictions where the sum over all topological sectors has been performed.

\subsection{Matching lattice QCD with the chiral effective theory}

When matching lattice results with the chiral effective theory, one should ideally first perform a continuum extrapolation in order to eliminate lattice artifacts. Since this is still not always feasible, an alternative is to incorporate discretization effects in the chiral effective theory by using the Symanzik approach [25]. 
From this point of view, the advantage of using GW fermions appears again: due to exact chiral symmetry at finite lattice spacing, lattice results are described by continuum chiral effective theory. The only effect of the descretization would be an $O\left(a^{2}\right)$ dependence of the LECs extracted from the matching ${ }^{1}$.

For Wilson-like fermions the situation is different: there will be new LECs associated to operators describing the lattice artifacts. This has been formulated systematically for pure Wilson fermions (WChPT) and Wilson Twisted Mass fermions [26, 27, 28]. This framework has been recently extended also to the $\varepsilon$ - expansion $[29,30]$. In the Wilson lattice formulation, lattice effects contribute to the explicit breaking of the chiral symmetry: the important issue is the relative power counting between the quark mass and the lattice spacing $a$. It has been observed that in the region $m \sim a \Lambda_{Q C D}^{2}$ (called GSM regime, with $a \sim O\left(\varepsilon^{4}\right)$ ), the explicit breaking of the chiral symmetry is still dominated by the quark mass, and for meson two-point functions lattice effects show up only at NNLO. On the other hand, when $m \sim a^{2} \Lambda_{Q C D}^{3}$ (Aoki regime, $a \sim O\left(\varepsilon^{2}\right)$ ) lattice effects contribute already at LO, leading to substantial changes of the continuum behavior. One can moreover define an intermediate regime, called GSM ${ }^{*}$, corresponding to the power counting $m \sim a^{4 / 3} \Lambda_{Q C D}^{7 / 3}$ $\left(a \sim O\left(\varepsilon^{3}\right)\right.$ ), where lattice artifacts appear at NLO. When simulating lattice QCD in the $\varepsilon$-regime with Wilson-like fermions, it is important to stay in the GSM-GSM* region, where lattice artifacts are suppressed: the small quark mass region must be approached by keeping also a small lattice spacing. This is an issue which can be addressed only a posteriori by analyzing lattice data using WChPT as a guidance.

\section{Results}

\subsection{LECs from spectral observables}

The link between the spectrum of the QCD Dirac operator and the spontaneous breaking of chiral symmetry is provided by the Banks-Casher relation [31],

$$
\lim _{\lambda \rightarrow 0} \lim _{m \rightarrow 0} \lim _{V \rightarrow \infty} \rho(\lambda, m)=\frac{\Sigma}{\pi} .
$$

Here $\rho(\lambda, m)$ represents the spectral density associated to the Euclidean massless Dirac operator, with imaginary eigenvalues $\left(i \lambda_{k}\right)$

$$
\rho(\lambda, m)=\frac{1}{V} \sum_{k=1}^{\infty}\left\langle\delta\left(\lambda-\lambda_{k}\right)\right\rangle .
$$

The spectral density can be computed within the chiral perturbation theory [32, 33, 34]. At LO in the $\varepsilon$-expansion, one can show that the partition function Eq. (1.5) at fixed topology is equivalent to the one of a Chiral Random Matrix Theory (RMT) [35, 36, 37]. It follows that RMT reproduces the same spectral density in terms of dimensionless variables $\zeta=\lambda \Sigma V, \mu=m \Sigma V$ (microscopic spectral density). While the chiral effective theory does not contain any information about the single eigenvalues, the advantage of this equivalence comes from the fact that with RMT one can extract the probability distributions of single eigenvalues $p_{k}\left(\zeta_{k}, \mu\right)[38,39]$. This means that the

\footnotetext{
${ }^{1}$ Additional operators related to the breaking of Euclidean symmetry can appear, but only at very high order.
} 
low-lying spectrum of the Dirac operator $\left\langle\lambda_{k}\right\rangle^{Q C D}(m)$ can be matched with the RMT expectation values

$$
\left\langle\zeta_{k}\right\rangle^{R M T}(\mu)=\int d \zeta_{k} p_{k}\left(\zeta_{k}, \mu\right) \zeta_{k}
$$

in order to extract the chiral condensate $\Sigma$. In particular, one matches

$$
\left\langle\lambda_{k}\right\rangle^{Q C D} \Sigma V=\left\langle\zeta_{k}\right\rangle^{R M T}
$$

Moreover, ratios of eigenvalues are parameter-free predictions which can be compared directly with lattice QCD results in order to check to which extent is RMT reproduced. Previous quenched studies with Neuberger fermions [40] observed a good agreement of the ratios involving lowest eigenvalues for lattice extents $L \gtrsim 1.5 \mathrm{fm}$.

This technique to extract $\Sigma$ has been adopted by many authors, using different implementations of GW fermions. DeGrand and collaborators [41] used Neuberger $N_{f}=2$ Dirac operator, with $a \simeq 0.15 \mathrm{fm}, L \simeq 1.5 \mathrm{fm}, m \Sigma V \simeq 2-5$ and topology fixed to $v=0,1$. They obtain the chiral condensate $\Sigma^{\overline{\mathrm{MS}}}(2 \mathrm{GeV})=(282(10) \mathrm{MeV})^{3}$.

JLQCD/TWQCD [42] also used $N_{f}=2$ Neuberger fermions, on a lattice with $a \simeq 0.11 \mathrm{fm}, L \simeq 1.78$ $\mathrm{fm}$ and with a quark mass corresponding to $\mu=m \Sigma V \simeq 0.556$. They fixed the topology to $v=0$. As final result for the quark condensate they quote $\Sigma^{\overline{\mathrm{MS}}}(2 \mathrm{GeV})=(251(7)(11) \mathrm{MeV})^{3}$. The first error is statistical, the second represents a systematic uncertainty, estimated by assuming that higher order corrections are the same as in the chiral effective theory.

P. Hasenfratz et al [43] implemented the $N_{f}=2+1$ parametrized fixed-point Dirac operator which satisfies the GW relation approximately. They adopted a lattice with $a \simeq 0.13 \mathrm{fm}, L \simeq 1.6 \mathrm{fm}$, $m_{u, d} \Sigma V \simeq 1.4$ and $m_{s} \Sigma V \simeq 12.3$; the topological charge was fixed to $v=0,1,2$. For the 2-flavor condensate they obtained $\Sigma^{\overline{\mathrm{MS}}}(2 \mathrm{GeV})=(239(11) \mathrm{MeV})^{3}$; this result is already corrected for higher order finite-size effects estimated through the chiral effective theory.

The RMT framework can be extended by adding an imaginary chemical potential, such that the spectrum of the Dirac operator is sensitive also to the pseudoscalar decay constant $F$ at LO [44]. This strategy has been adopted by DeGrand and collaborators [45], yielding $\Sigma^{\overline{\mathrm{MS}}}(2 \mathrm{GeV})=$ $(234(4) \mathrm{MeV})^{3}$ and $F=84(5) \mathrm{MeV}$ from a lattice with $a \simeq 0.13 \mathrm{fm}, L \simeq 1.6 \mathrm{fm}$ and $\mu \simeq 4.4$.

The problematic point of this approach is that while higher order finite-size corrections can be computed within the chiral effective theory, this is not possible in the framework of RMT. This means that systematic errors on $\Sigma, F$ might be difficult to quantify.

In another recent work [46] it is shown that the quark condensate can be extracted from suitable (renormalizable) spectral observables, for instance the number of Dirac operator modes contained in a given interval. The technique has been used on Wilson lattice QCD in the p-regime, but could be investigated also in the $\varepsilon$-regime. The advantage of this method is that those observables can be computed in the chiral effective theory: higher order corrections can be computed and systematic effects of the extracted LECs can be estimated.

In relation to this, in [47] the quark condensate and the spectral density of the Dirac operator at fixed topological charge have been computed in the chiral effective theory by using a technique which is able to smoothly connect $p$ - and $\varepsilon$ - regimes. S. Hashimoto presented at this conference preliminary lattice results for the spectral density obtained by the JLQCD/TWQCD with $N_{f}=2+1$ 
(Neuberger fermions) [48]. Through the matching with the NLO predictions of [47], the LECs $\Sigma$, $F$ and $L_{6}$ are extracted.

\subsection{LECs from two-point meson correlators}

Meson two-point functions in the $\varepsilon$-expansion have been computed for the first time by Hansen [49] at NNLO. Here we report as example the pseudoscalar density and axial current time correlators at NLO, which is usually the level adopted in the matching with lattice results. For the full dynamical case, NLO correlators have the general form

$$
\begin{aligned}
& C_{P}^{a b}(t)=\int d^{3} \vec{x}\left\langle P^{a}(x) P^{b}(0)\right\rangle=\delta^{a b}\left[a_{P}+b_{P} h_{1}(t / T)\right], \\
& C_{A}^{a b}(t)=\int d^{3} \vec{x}\left\langle A_{0}^{a}(x) A_{0}^{b}(0)\right\rangle=\delta^{a b}\left[a_{A}+b_{A} h_{1}(t / T)\right],
\end{aligned}
$$

with a parabolic time dependence given by the function

$$
h_{1}(\tau)=\frac{1}{2}\left[\left(|\tau|-\frac{1}{2}\right)^{2}-\frac{1}{12}\right] .
$$

The coefficients $a_{P, A}, b_{P, A}$ will in general contain non-perturbative integrals over the zero modes, and will be functions only of the volume and the LO LECs $\Sigma$ and $F$. In particular, for $N_{f}=2$ one obtains, after performing the integrals and summing over all topological sectors

$$
\begin{aligned}
& a_{P}=\frac{L^{3} \Sigma_{\text {eff }}^{2}}{\mu_{\text {eff }}} \frac{I_{2}\left(2 \mu_{\mathrm{eff}}\right)}{I_{1}\left(2 \mu_{\mathrm{eff}}\right)}, \quad b_{P}=\frac{T \Sigma^{2}}{2 F^{2}}\left(2-\frac{1}{\mu} \frac{I_{2}(2 \mu)}{I_{1}(2 \mu)}\right), \\
& a_{A}=-\frac{F^{2}}{T}\left(1-\frac{I_{2}\left(2 \mu_{\mathrm{eff}}\right)}{\mu_{\mathrm{eff}} I_{1}\left(2 \mu_{\mathrm{eff}}\right)}\right)-\frac{2 \beta_{1}}{T \sqrt{V}}\left(1-\frac{I_{2}(2 \mu)}{\mu I_{1}(2 \mu)}\right)+\frac{2 T k_{00}}{V} \frac{I_{2}(2 \mu)}{\mu I_{1}(2 \mu)}, \\
& b_{A}=-\frac{2 T}{V} \frac{I_{2}(2 \mu)}{\mu I_{1}(2 \mu)} .
\end{aligned}
$$

We have introduced $\mu_{\text {eff }}=m \Sigma_{\text {eff }} V$, where $\Sigma_{\text {eff }}$ is the one-loop chiral condensate [3]

$$
\Sigma_{\mathrm{eff}}=\Sigma\left(1+\frac{3}{2 F^{2}} \frac{\beta_{1}}{\sqrt{V}}\right) \text {. }
$$

$\beta_{1}$ and $k_{00}$ are so-called shape coefficients, and depend only on the geometry of the box [50].

Meson correlators at NLO in the $\varepsilon$-expansion have been computed also in the quenched or partially quenched case, and in fixed topology sectors [34, 51, 52]. In [53] the so-called mixed regime has been considered, where some quarks are in the $\varepsilon$-regime and others are in the $p$-regime.

In [30, 29], $O\left(a^{2}\right)$ effects have been computed in WChPT at NLO in the $\mathrm{GSM}^{*}$ regime for $N_{f}=$ 2. They are parametrized by an extra LEC $c_{2}$, which is discretization-dependent, and contribute to the constant terms $a_{P, A}$ in Eqs. $(3.8,3.9)$,

$$
a_{P, a^{2}}=a_{P}+\rho \frac{L^{3} \Sigma^{2} \Delta_{a^{2}}}{2}, \quad a_{A, a^{2}}=a_{A}+\rho \frac{F^{2} \Delta_{a^{2}}}{T},
$$

where

$$
\Delta_{a^{2}}=\frac{4 \tilde{\mu}^{2} I_{1}^{3}(2 \tilde{\mu})-11 \tilde{\mu} I_{1}^{2}(2 \tilde{\mu}) I_{2}(2 \tilde{\mu})+2\left(3-2 \tilde{\mu}^{2}\right) I_{1}(2 \tilde{\mu}) I_{2}^{2}(2 \tilde{\mu})+5 \tilde{\mu} I_{2}^{3}(2 \tilde{\mu})}{2 \tilde{\mu}^{3} I_{1}^{2}(2 \tilde{\mu}) I_{2}(2 \tilde{\mu})} .
$$



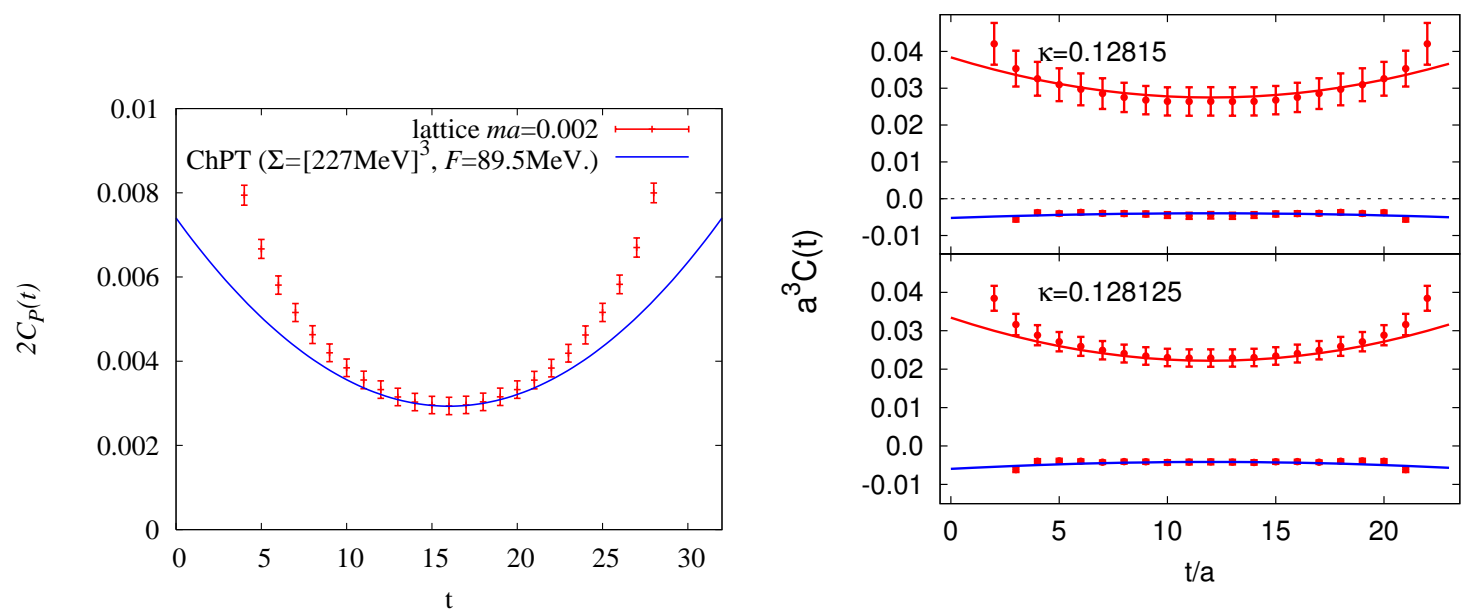

Figure 1: Left side: time dependence of the pseudoscalar correlator computed by [54]. Right side: pseudoscalar and axial correlators computed in [22], for $L \simeq 2.8 \mathrm{fm}$ and two quark mass values $(\kappa=$ $0.12815,0.128125$, corresponding to $\tilde{\mu} \simeq 2.1,2.7$ ). Here the axial vector correlator is multiplied by a factor 50 for better visibility.

We have introduced $\tilde{\mu}=m_{\mathrm{PCAC}} \Sigma V$ and the dimensionless parameter $\rho \equiv F^{2} c_{2} a^{2} V$. In the continuum part, the replacement $m \rightarrow m_{\mathrm{PCAC}}(\mu \rightarrow \tilde{\mu})$ is understood.

JLQCD computed the scalar, pseudoscalar, vector and axial-vector correlators using Neuberger fermions, $N_{f}=2, a \simeq 0.11 \mathrm{fm}, L \simeq 1.78 \mathrm{fm}, \mu \simeq 0.556$ and topology $v=0$ [54]. By simultaneously matching the pseudoscalar and the axial with continuum chiral effective theory at NLO, they obtained $\Sigma^{\overline{\mathrm{MS}}}(2 \mathrm{GeV})=(239.8(4.0) \mathrm{MeV})^{3}$ and $F=87.3(5.6) \mathrm{MeV}$. On the left side of Fig. 1 we report the pseudoscalar correlator.

In [22] pseudoscalar and axial 2-point functions in the $\varepsilon$-regime have been computed with $N_{f}=2$ NHYP Wilson fermions, using the reweighting technique proposed in [20]. The lattice spacing is fixed to $a \simeq 0.115 \mathrm{fm}$; two volumes have been considered, $L \simeq 1.84 \mathrm{fm}$ with a mass range $\tilde{\mu}=m_{\mathrm{PCAC}} \Sigma V \simeq 0.7-2.9$ and $L \simeq 2.8 \mathrm{fm}$ with $\tilde{\mu} \simeq 2.1-5$. From a continuum NLO fit of the large volume data, they obtained $\Sigma^{\overline{\mathrm{MS}}}(2 \mathrm{GeV})=(248(6) \mathrm{MeV})^{3}$ and $F=90(4) \mathrm{fm}$. On the right side of Fig.1 the two correlators for the two lightest quark masses are shown. In [30] the data have been reanalyzed including the $O\left(a^{2}\right)$ calculated in the $\mathrm{GSM}^{*}$ regime, Eqs. (3.12,3.13), yielding $\Sigma^{\overline{\mathrm{MS}}}(2 \mathrm{GeV})=(249(4) \mathrm{MeV})^{3}, F=88(3) \mathrm{MeV}$ and $c_{2}=0.02(8) \mathrm{GeV}^{4}$. This result shows that cut-off effects do not impact the extraction of the LECs beyond the level of the statistical uncertainties.

\section{Conclusions}

Lattice simulations in the $\varepsilon$-regime can give important informations about properties of QCD at low energy. In particular, they can be used to extract Low-Energy-Couplings of the chiral effective theory: these determinations are independent from the ones obtained in the usual infinite volume case and are affected by different systematic uncertainties.

Computations in the $\varepsilon$-regime are numerically still very challenging, especially if one uses lattice discretizations which preserve the chiral symmetry at finite lattice spacing. Recently it has been 
shown that simulations with Wilson-like fermions are feasible in the $\varepsilon$-regime: they are more affordable from the point of view of the numerical cost, but the explicit breaking of chiral symmetry requires a special attention when matching lattice results with the chiral effective theory.

In general, it would be very useful to perform lattice computations in a wide range of volumes and lattice spacings, in order to check if the predicted NLO finite-size scaling is verified and to have a reliable estimation of systematic errors.

There are several other applications of the chiral $\varepsilon$-expansion in combination with lattice calculations. For instance, the theoretical predictions obtained in the mixed regime introduced in [53] can be matched with lattice results: a possible setup could be to use Wilson sea quarks in the $p$ regime and $\mathrm{GW}$ valence quarks in the $\varepsilon$-regime.

Another possible application is to consider heavy-light mesons and treat the light quarks in the $\varepsilon$-expansion, in particular within the framework of Heavy Meson Chiral Perturbation Theory.

In [55], suitable correlators are defined both in the $p$ and in the $\varepsilon$-regime to extract the couplings of the effective weak Hamiltonian which parametrize the $K \rightarrow \pi \pi$ amplitudes. This strategy has been applied to quenched Neuberger fermions [56], and could be adopted also in the dynamical case.

\section{References}

[1] S. Necco, PoS CONFINEMENT8 (2008) 024, 0901.4257.

[2] J. Gasser and H. Leutwyler, Phys. Lett. B184 (1987) 83.

[3] J. Gasser and H. Leutwyler, Phys. Lett. B188 (1987) 477.

[4] H. Leutwyler and A. Smilga, Phys. Rev. D46 (1992) 5607.

[5] P.H. Ginsparg and K.G. Wilson, Phys. Rev. D25 (1982) 2649.

[6] M. Lüscher, Phys. Lett. B428 (1998) 342, hep-lat/9802011.

[7] H. Neuberger, Phys. Lett. B417 (1998) 141, hep-lat/9707022.

[8] D.B. Kaplan, Phys. Lett. B288 (1992) 342, hep-lat/9206013.

[9] P. Hasenfratz, V. Laliena and F. Niedermayer, Phys. Lett. B427 (1998) 125, hep-lat/9801021.

[10] P. Hernández, K. Jansen and L. Lellouch, Phys. Lett. B469 (1999) 198, hep-lat/9907022.

[11] MILC, T.A. DeGrand, Phys. Rev. D64 (2001) 117501, hep-lat/0107014.

[12] P. Hasenfratz et al., Nucl. Phys. B643 (2002) 280, hep-lat/0205010.

[13] W. Bietenholz et al., JHEP 02 (2004) 023, hep-lat/0311012.

[14] L. Giusti et al., JHEP 04 (2004) 013, hep-lat/0402002.

[15] H. Fukaya, S. Hashimoto and K. Ogawa, Prog. Theor. Phys. 114 (2005) 451, hep-lat/0504018.

[16] W. Bietenholz and S. Shcheredin, Nucl. Phys. B754 (2006) 17, hep-lat/0605013.

[17] L. Giusti and S. Necco, JHEP 04 (2007) 090, hep-lat/0702013.

[18] L. Del Debbio et al., JHEP 02 (2006) 011, hep-lat/0512021.

[19] L. Del Debbio et al., JHEP 02 (2007) 056, hep-lat/0610059.

[20] A. Hasenfratz, R. Hoffmann and S. Schaefer, Phys. Rev. D78 (2008) 014515, 0805.2369. 
[21] M. Lüscher and F. Palombi, (2008), 0810.0946.

[22] A. Hasenfratz, R. Hoffmann and S. Schaefer, Phys. Rev. D78 (2008) 054511, 0806.4586.

[23] K. Jansen et al., PoS LAT2007 (2007) 084, 0711.1871.

[24] K. Jansen, A. Nube and A. Shindler, (2008), 0810.0300.

[25] K. Symanzik, Nucl. Phys. B226 (1983) 187; K. Symanzik, Nucl. Phys. B226 (1983) 205.

[26] S.R. Sharpe and J. Singleton, Robert L., Phys. Rev. D58 (1998) 074501, hep-lat/9804028.

[27] G. Rupak and N. Shoresh, Phys. Rev. D66 (2002) 054503, hep-lat/0201019.

[28] S.R. Sharpe and J.M.S. Wu, Phys. Rev. D70 (2004) 094029, hep-lat/0407025.

[29] A. Shindler, Phys. Lett. B672 (2009) 82, 0812.2251.

[30] O. Bär, S. Necco and S. Schaefer, JHEP 03 (2009) 006, 0812.2403.

[31] T. Banks and A. Casher, Nucl. Phys. B169 (1980) 103.

[32] A.V. Smilga and J. Stern, Phys. Lett. B318 (1993) 531.

[33] J.C. Osborn, D. Toublan and J.J.M. Verbaarschot, Nucl. Phys. B540 (1999) 317, hep-th/9806110.

[34] P.H. Damgaard et al., Nucl. Phys. B547 (1999) 305, hep-th/9811212.

[35] E.V. Shuryak and J.J.M. Verbaarschot, Nucl. Phys. A560 (1993) 306, hep-th/9212088.

[36] J.J.M. Verbaarschot and I. Zahed, Phys. Rev. Lett. 70 (1993) 3852, hep-th/9303012.

[37] J.J.M. Verbaarschot, Phys. Rev. Lett. 72 (1994) 2531, hep-th/9401059.

[38] S.M. Nishigaki, P.H. Damgaard and T. Wettig, Phys. Rev. D58 (1998) 087704, hep-th/9803007.

[39] P.H. Damgaard and S.M. Nishigaki, Phys. Rev. D63 (2001) 045012, hep-th/0006111.

[40] L. Giusti et al., JHEP 11 (2003) 023, hep-lat/0309189.

[41] T. DeGrand, Z. Liu and S. Schaefer, Phys. Rev. D74 (2006) 094504, hep-lat/0608019.

[42] H. Fukaya et al., Phys. Rev. D76 (2007) 054503, 0705.3322.

[43] P. Hasenfratz et al., (2007), 0707.0071.

[44] G. Akemann et al., Nucl. Phys. B766 (2007) 34, hep-th/0609059.

[45] T. DeGrand and S. Schaefer, Phys. Rev. D76 (2007) 094509, 0708.1731.

[46] L. Giusti and M. Lüscher, JHEP 03 (2009) 013, 0812.3638.

[47] P.H. Damgaard and H. Fukaya, JHEP 01 (2009) 052, 0812.2797.

[48] S. Hashimoto, PoS CD09 (2009) 004.

[49] F.C. Hansen, Nucl. Phys. B345 (1990) 685.

[50] P. Hasenfratz and H. Leutwyler, Nucl. Phys. B343 (1990) 241.

[51] P.H. Damgaard et al., Nucl. Phys. B629 (2002) 445, hep-lat/0112016.

[52] P.H. Damgaard et al., Nucl. Phys. B656 (2003) 226, hep-lat/0211020.

[53] F. Bernardoni et al., JHEP 10 (2008) 008, 0808.1986.

[54] JLQCD, H. Fukaya et al., Phys. Rev. D77 (2008) 074503, 0711.4965.

[55] L. Giusti et al., JHEP 11 (2004) 016, hep-lat/0407007.

[56] L. Giusti et al., Phys. Rev. Lett. 98 (2007) 082003, hep-ph/0607220. 